\title{
ГИПЕРБОЛИЧЕСКИЕ МОДЕЛИ В МЕХАНИКЕ ГЕТЕРОГЕННЫХ СРЕД
}

B.C. Суров

В рамках многоскоростной модели гетерогенной среды с газодинамическим ядром численно исследована динамика акустических латентных волн в воздушном тумане.

Ключевые слова: многоскоростная многокомпонентная среда, гиперболические системы уравнений, латентные волны, численное моделирование.

В гетерогенных средах помимо известных из однофазной газодинамики волн вида ударных (сжатия), в которых уровень давления растет, разрежения, в которых давление, напротив, уменьшается, существуют так называемые латентные волны, характеризуемые измененными значениями объемных долей отдельных компонентов в смеси и их локальных скоростей при неизменном профиле давления. Отслеживать латентные волны затруднительно, поскольку гетерогенные среды могут быть оптически непрозрачными, а традиционным способом, - по изменению давления, - зафиксировать их невозможно. При падении латентной волны на упругую преграду, например, в запыленном газе деформация преграды происходит за счет передачи аккумулированного в волне количества движения, связанного с дисперсной фракцией. Очевидно, что динамическое действие латентной волны на преграду зависит от ее ориентации по отношению к потоку в волне и это принципиально отличает ее от газодинамической волны.

Эффективным инструментом исследования латентных волн является компьютерное моделирование. В настоящей работе численно исследуется динамика «коротких» латентных волн в газожидкостной смеси при их 
взаимодействии с жесткими преградами, а также друг с другом. Моделирование проводилось в рамках гиперболической модели с газодинамическим ядром из [1]. В этой модели введено в рассмотрение такое состояние среды как смесь в целом, характеризуемое осредненными значениями величин, уравнения для которых совпадают с газодинамическими. К этим соотношениям добавляются уравнения, выражающие законы сохранения, но только для тех компонентов смеси, в которых локальные скорости перемещения возмущений не превышают скорость движения волны в смеси в целом. При этом считалось, что остальные волны поглощаются средой, формируя волну в смеси в целом. Давление полагалось общим для всех фракций смеси. Для газожидкостных смесей модель с газодинамическим ядром удовлетворительно описывает волновые процессы, наблюдаемые в экспериментах (см. [2]).

Существование латентных волн подтверждается, например, опытами Тиндаля [3], а также наблюдениями автора статьи. В этих опытах отмечалось появление «эха» при распространении звукового сигнала в тумане, что связано с расщеплением исходного импульса на «быстрый» и «медленный». Последний представляет собой латентную волну с измененным уровнем концентрации дисперсной фракции в смеси. Отметим также, что если для воспроизведения опытов Тиндаля вместо модели из [1] привлечь другие модели, то получим результаты, противоречащие эксперименту. Так в случае применения модели Baer'a-Nunziato [4] или Saurel'aAbgrall'a [5], скорости «расщепленной» волны оказываются равными 340 и $1500 \mathrm{~m} / \mathrm{c}$, что существенно выше экспериментальных значений. Аналогичные результаты получаются при использовании модели В.Ф. Куропатенко [6], а также модели из работы [7]. Заметим, что впервые эффект расщепления импульса в многокомпонентных средах на волны, распространяющиеся с различными скоростями, был теоретически предсказан в работе [8].

Модель среды и результаты численного эксперимента. В работе использовалась модель $n$-компонентной гетерогенной среды с газодинамическим ядром из [1], уравнения которой для бинарной смеси, состоящей из идеального газа с показателем адиабаты $\gamma$ и несжимаемой фракции, принимают вид:

$$
\begin{gathered}
\frac{\partial \rho}{\partial t}+u \frac{\partial \rho}{\partial x}+\rho \frac{\partial u}{\partial x}=0, \frac{\partial u}{\partial t}+u \frac{\partial u}{\partial x}+\frac{1}{\rho} \frac{\partial p}{\partial x}=0 \\
\frac{\partial p}{\partial t}+u \frac{\partial p}{\partial x}-c^{2}\left(\frac{\partial \rho}{\partial t}+u \frac{\partial \rho}{\partial x}\right)=0 \\
\frac{\partial \alpha_{\mathrm{s}}}{\partial t}+u_{\mathrm{s}} \frac{\partial \alpha_{\mathrm{s}}}{\partial x}+\alpha_{\mathrm{s}} \frac{\partial u_{\mathrm{s}}}{\partial x}=0, \frac{\partial u_{\mathrm{s}}}{\partial t}+u_{\mathrm{s}} \frac{\partial u_{\mathrm{s}}}{\partial x}+\frac{c_{\mathrm{s}}^{2}}{\alpha_{\mathrm{s}}} \frac{\partial \alpha_{\mathrm{s}}}{\partial x}+\frac{1}{\rho_{\mathrm{s}}} \frac{\partial p}{\partial x}=\frac{\lambda_{\mathrm{s}}\left(u-u_{\mathrm{s}}\right)}{\alpha_{\mathrm{s}} \rho_{\mathrm{s}}},
\end{gathered}
$$


где $p$ - давление, $t$ - время, $u$ - скорость смеси, $x$ - пространственная переменная, $\alpha_{\mathrm{s}}-$ объемная доля дисперсной фракции в смеси, $\lambda_{\mathrm{s}}-$ коэффициент сопротивления, $u_{\mathrm{s}}-$ скорость дисперсной фракции, $\rho=\alpha_{\mathrm{s}} \rho_{\mathrm{s}}+\left(1-\alpha_{\mathrm{s}}\right) \rho_{\mathrm{g}}^{0}-$ плотность смеси, $\rho_{\mathrm{s}}$ - плотность несжимаемой компоненты.

Газодинамическая скорость звука и скорость перемещения латентной волны определяются в соответствии с формулами (см. [1]):

$$
c=\sqrt{\frac{\gamma p}{\left(1-\alpha_{\mathrm{s}}\right) \rho}}, \quad c_{\mathrm{s}}=\sqrt{\frac{p}{\rho_{\mathrm{s}}}} .
$$
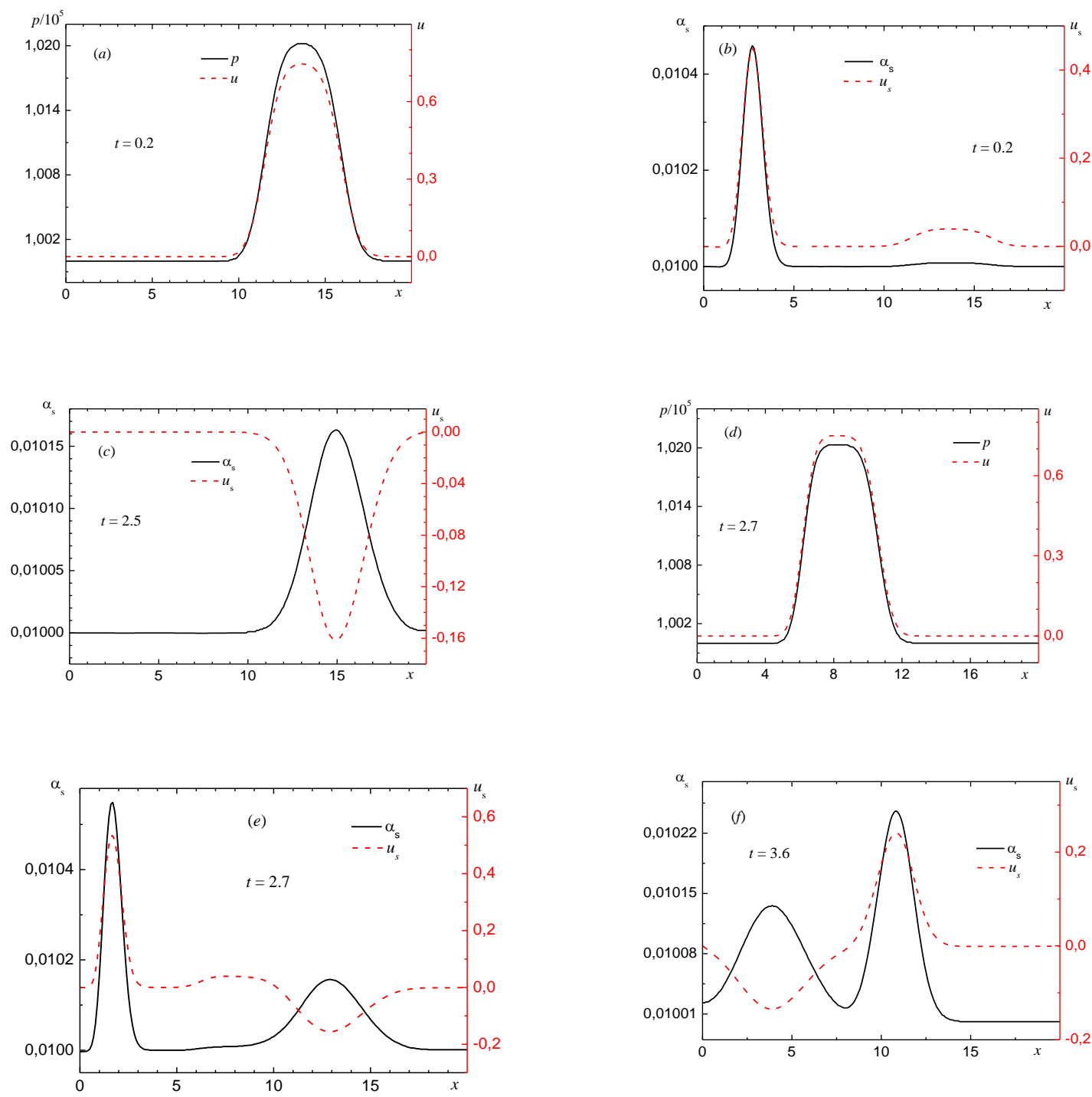

Рис. 1. Эволюция газодинамических и латентных волн, генерируемых левой подвижной стенкой при $\lambda_{\mathrm{s}}=0$ в моменты времени $t=0,3,2,5,2,7$ и 3,6 с $(a-f)$

Наглядную картину динамики латентных волн дают расчеты для коротких импульсов. Для численного интегрирования (1) применялся узловой 
метод характеристик (УМХ), описание которого имеется в [9]. С использованием УМХ рассчитана задача о распространении «короткого» акустического импульса давления по смеси, генерируемого смещающейся с постоянной скоростью до момента времени $T$ левой непроницаемой для смеси границы:

$$
x_{0}(t)=\left\{\begin{array}{lll}
A t, & \text { если } t \leq T, \\
A T, & \text { если } t>T,
\end{array}\right.
$$

где $A=0,05 \mathrm{м}, T=0,084$ с. Плотность жидкой фракции $\rho_{\mathrm{s}}=1000 \mathrm{\kappa г} / \mathrm{m}^{3}$, показатель адиабаты газа $\gamma=1,4$, его плотность в невозмущенном состоянии $\rho_{\mathrm{g}(0)}^{0}=1,19 \kappa \Gamma / \mathrm{M}^{3}$. Коэффициент сопротивления $\lambda_{\mathrm{s}}$ принимался равным нулю и

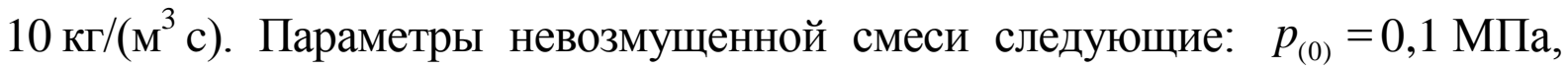
$u_{(0)}=u_{\mathrm{s}(0)}=0, \alpha_{\mathrm{s}(0)}=0,05$. $\mathrm{B}$ расчетах использовалась равномерная сетка с шагом $\Delta x=0,1 \mathrm{м}$.

На рис. $1 a-f$ представлены результаты вычислений, полученные к моментам времени $t=0,3,2,5,2,7$ и 3,6 с при $\lambda_{\mathrm{s}}=0$. На правой границе расчетной области ставилось «мягкое» граничное условие свободного истечения, поэтому газодинамический импульс повышенного давления, перемещающийся со скоростью рассчитываемой по первой формуле (2), беспрепятственно выходит за пределы расчетной области. Момент выхода этой волны приведен на рис. $1 a-b$. Латентная волна, движущаяся с более низкой скоростью, определяемой второй формулой (2), трансформируется в треугольного вида импульс (рис. $1 b$ ). При подходе латентной волны к правой границе, граничное условие на которой было изменено на тип «непроницаемая» стенка, поэтому по ее достижению импульс отражается, что видно из рис. $1 c$. В момент времени $t=2,5$ с левая граница вновь приводилась в движение по закону (3). Характеристики сформировавшихся газодинамической и латентной волн к моменту $t=2,7$ с представлены на рис. $1 d-e$. Как и ранее, газодинамическая волна «выпускалась» за пределы расчетной области путем смены типа граничного условия на правой границе. В дальнейшем происходит взаимодействие двух латентных волн друг с другом, заключительная фаза которого к моменту $t=3,6$ с приведена на рис. $1 f$, когда эти две волны расходятся в противоположных направлениях.

На рис. $2 a-d$ представлены результаты вычислений, полученные к моментам времени $t=0,3,1,6$ и 2,5 с при $\lambda_{\mathrm{s}}=10$ кг $/\left(\mathrm{M}^{3} \mathrm{c}\right)$. Параметры среды те же, что и в первой задаче. Как и ранее импульс генерировался приведением в движение по закону (3) левой непроницаемой для смеси границы (рис. $2 a-c)$. Граничное условие на правой границе, как и ранее, моделировало условие свободного истечения, но после выхода газодинамической волны за пределы расчетной области заменялось на «жесткую» стенку, так что латентная волна отражалась этой границы (рис. $2 d$ ). Из рис. $2 a-d$ видно, при учете сил сопротивления латентный импульс становится асиммет- 
ричным. Кроме того, из данных на рис. 1-2 следует, что латентная волна с течением времени «размывается»- ее нижнее основание увеличивается, амплитуда же, напротив, уменьшается но все это происходит на фоне неизменного профиля давления.
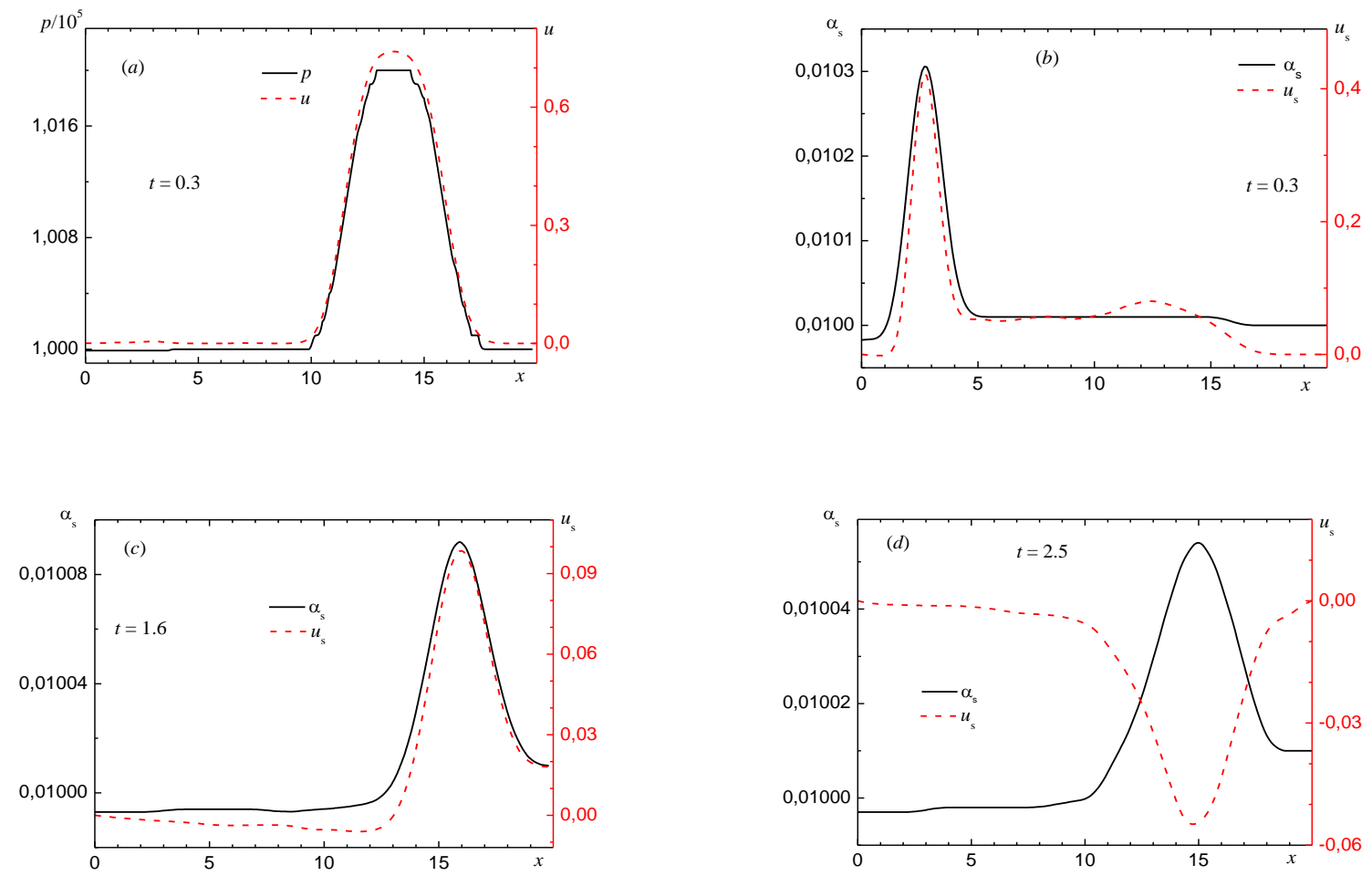

Рис. 2. Эволюция газодинамического и латентного скачка, генерируемых левой

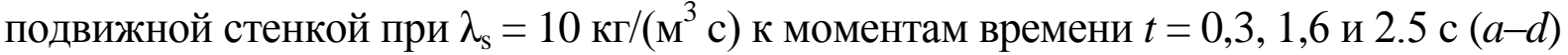

Заключение. В рамках многоскоростной модели гетерогенной среды с газодинамическим ядром численно исследована динамика латентных волн в газожидкостной смеси - их отражение от твердых границ, взаимодействие друг с другом. Показано, что расщепление звукового сигнала на газодинамический и латентный импульс объясняет возникновение «эха» в туманных средах, наблюдаемое в экспериментах Тиндаля.

Библиографический список

1. Суров, В.С. Гиперболическая модель многоскоростной гетерогенной среды / В.С. Суров // Инженерно-физический журн. - 2012. - Т. 85. - № 3. C. 495-502.

2. Суров, В.С. Гиперболические модели в механике гетерогенных сред / В.С. Суров // Журн. Вычисл. Математ. и Матем. Физики. - 2014. - Т. 54. № 1. - C. 139-149. 
3. Стрэтт, Дж.В. Теория звука. Т. 2. / Дж.В. Стрэтт (Лорд Рэлей). - Москва: ГИТТЛ. - 1944.

4. Baer, M. A two-phase mixture theory for deflagration-to-detonation transition (DDT) in reactive granular materials / M. Baer, J. Nunziato // Int. J. Multiphase Flow. - 1986. - V.12. - Pp. 861-889.

5. Zein, A. Modeling phase transition for compressible two-phase flows applied to metastable liquids / A. Zein, M. Hantke, G. Warnecke // J. Comput. Phys. - 2010. - V. 229. - Pp. 2964-2998.

6. Куропатенко, В.Ф. Модель многокомпонентной среды / В.Ф. Куропатенко // Докл. РАН. - 2005. - Т. 403. - № 6. - С. 761-763.

7. Yeom, G.-S. A modified HLLC-type Riemann solver for the compressible six-equation two-fluid model / G.-S. Yeom, K.-S. Chang // Computers \& Fluids. 2013. - V. 76. - Pp. 86-104.

8. Клейман, Я.З. О распространении волн слабого разрыва в многокомпонентной среде / Я.З. Клейман // Акуст. журн. - 1958. - Т. 4. - № 3. C. 253-262.

9. Суров, В.С. Узловой метод характеристик для расчета течений многоскоростной гетерогенной среды / В.С. Суров // Инженерно-физический журн. - 2013. - Т. 86. - № 5. - С. 1088-1096. 